\title{
Melnikov's method and Arnold diffusion for perturbations of integrable Hamiltonian systems ${ }^{\text {a) }}$
}

\author{
Philip J. Holmes \\ Department of Theoretical and Applied Mechanics, Cornell University, Ithaca, New York 14853 \\ Jerrold E. Marsden \\ Department of Mathematics, University of California, Berkeley, California 94720
}

(Received 17 June 1981; accepted for publication 23 October 1981)

We start with an unperturbed system containing a homoclinic orbit and at least two families of periodic orbits associated with action angle coordinates. We use Kolmogorov-Arnold-Moser (KAM) theory to show that some of the resulting tori persist under small perturbations and use a vector of Melnikov integrals to show that, under suitable hypotheses, their stable and unstable manifolds intersect transversely. This transverse intersection is ultimately responsible for Arnold diffusion on each energy surface. The method is applied to a pendulum-oscillator system.

PACS numbers: $46.10 .+\mathrm{z}, 03.20 .+\mathrm{i}$

\section{INTRODUCTION}

In a previous paper (Holmes and Marsden) ${ }^{1}$ we developed a method for proving the existence of Smale horseshoes in two-degree-of-freedom Hamiltonian and nearly Hamiltonian systems. This paper extends those methods to systems with three or more degrees of freedom. We start with an unperturbed system containing a homoclinic orbit and at least two families of periodic orbits associated with action coordinates. We use KAM theory to show that some of the resulting tori persist under small perturbations and use a vector of Melnikov integrals to show that, under suitable hypotheses, their stable and unstable manifolds intersect transverselly. This transverse intersection is ultimately responsible for Arnold diffusion on each energy surface.

Our methods are a generalization of those of Arnold ${ }^{2}$ where "Arnold diffusion" was first introduced. The applications are, however, somewhat different and, we believe, of more direct physical interest.

For two-dimensional forced systems, the existence of transverse homoclinic orbits using Melnikov type methods is discussed in Chow, Hale, and Mallet-Paret, ${ }^{3}$ Holmes, ${ }^{4}$ and Greenspan and Holmes. ${ }^{5}$ These methods apply to two-degree-of-freedom Hamiltonian systems through the process of reduction and are discussed in Holmes and Marsden. ${ }^{1}$ These methods also apply to certain infinite-dimensional problems with external forcing and damping; see Holmes and Marsen. ${ }^{6}$ The purpose of this paper is to extend the Melnikov method to Hamiltonian systems with three or more degrees of freedom where the new phenomenon of Arnold diffusion arises.

The main example treated in this paper is a Hamiltonian system consisting of a pendulum coupled to two oscillators (with amplitude-dependent frequencies). The system is shown to have Arnold diffusion. Using the techniques in our previous paper, one can also show that the Arnold diffusion on a certain energy surface survives suitable positive and negative damping perturbations.

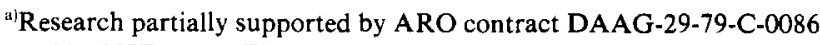
and by NSF grants ENG 78-02891 and MCS-78-06718.
We shall assume that our coordinates are given in canonical form. However, many interesting problems involving rigid body dynamics are best done in a more general Lie group theoretic context. This situation is discussed in Holmes and Marsden. ${ }^{7}$

\section{TRANSVERSAL INTERSECTION OF INVARIANT MANIFOLDS BY MELNIKOV'S METHOD}

In this section we are concerned with perturbations of Hamiltonian systems of the form

$$
H^{\circ}(q, p, x, y)=F(q, p)+G(x, y),
$$

where $(q, p, x, y)$ are canonical coordinates on a $2(n+1)$-dimensional symplectic manifold $P ; q$ and $p$ are real and $x=\left(x^{1}, \ldots, x^{n}\right), y=\left(y_{1}, \ldots, y_{n}\right)$. We assume the coordinates are canonical although, in some examples such as the rigid body, this requires modification (Holmes and Marsden ${ }^{7}$ ). We shall also assume that action-angle coordinates $\left(\theta_{1}, \ldots, \theta_{n}, I_{1}, \ldots, I_{n}\right)$ can be found in a certain region of phase space such that (2.1) takes the form

$$
H^{0}(q, p, \theta, I)=F(q, p)+\sum_{i=1}^{n} G_{i}\left(I_{i}\right)
$$

We also assume that $G_{i}(0)$ and that

$$
\Omega_{j}\left(I_{j}\right)=\frac{\partial G_{j}}{\partial I_{j}}>0 \text { for } I_{j}>0 .
$$

The perturbed problem we consider has the form

$$
H^{\epsilon}(q, p, \theta, I)=F(q, p)+\sum_{i=1}^{n} G_{i}\left(I_{i}\right)+\epsilon H^{1}(q, p, \theta, I),
$$

where $H^{1}$ is $2 \pi$-periodic in $\theta_{1}, \ldots, \theta_{n}$. Now we recall how this $(n+1)$-degree-of-freedom system may be reduced to an $n$ degree-of-freedeom nonautonomous system; the reader should refer to Holmes and Marsden ${ }^{1}$ for details.

Choose one of the action coordinates, say $I_{n}$. Since $\Omega_{n}\left(I_{n}\right)>0$ for $I_{n}>0$, we can invert the equation

$$
H^{\epsilon}(q, p, \theta, I)=h
$$

to obtain

$$
I_{n}=L^{\epsilon}\left(q, p, \theta_{1}, \ldots, \theta_{n}, I_{1}, \ldots, I_{n-1} ; h\right) .
$$


If we write

$$
L^{\epsilon}=L^{0}+\epsilon L^{1}+O\left(\epsilon^{2}\right),
$$

then a simple computation shows that

$$
\begin{aligned}
& L^{0}\left(q, p, I_{1}, \ldots, I_{n-1} ; h\right) \\
& \quad=G_{n}^{-1}\left(h-F(q, p)-\sum_{j=1}^{n-1} G_{j}\left(I_{j}\right)\right)
\end{aligned}
$$

and

$$
\begin{aligned}
& L^{1}\left(q, p, \theta_{1}, \ldots, \theta_{n}, I_{1}, \ldots, I_{n-1} ; h\right) \\
& =-\frac{H^{1}\left(q, p, \theta_{1}, \ldots, \theta_{n}, I_{1}, \ldots, I_{n-1}\right), L^{0}\left(q_{1}, p, I_{1}, \ldots, I_{n-1} ; h\right)}{\Omega_{n}\left(L^{0}\left(q, p, I_{1}, \ldots, I_{n-1} ; h\right)\right)} .
\end{aligned}
$$

Changing variables from $t$ to $\theta_{n}$ and writing ( $)^{\prime}$ for $\left(d / d \theta_{n}\right)$ ( ), Hamilton's equations for $H^{\epsilon}$ become

$$
\begin{aligned}
& q^{\prime}=-\frac{\partial L^{\epsilon}}{\partial p}, \quad p^{\prime}=\frac{\partial L^{\epsilon}}{\partial q}, \\
& \theta_{j}^{\prime}=-\frac{\partial L^{\epsilon}}{\partial I_{j}}, \quad I_{j}^{\prime}=-\frac{\partial L^{\epsilon}}{\partial \theta_{j}}, \quad j=1, \ldots, n-1 .
\end{aligned}
$$

Using (2.7)-(2.9), Eqs. (2.10) are in the form of a $2 \pi$-periodically forced $n$-degree-of-freedom Hamiltonian system. Notice that $L^{0}$ is (formally) completely integrable, having $n$ constants of the motion given by

$$
L^{0} \text { (energy) and }\left(I_{1}, \ldots, I_{n-1}\right)=\left(l_{1}, \ldots, l_{n-1}\right),
$$

or alternatively,

$$
L^{0} \text { and }\left(G_{1}\left(I_{1}\right), \ldots, G_{n-1}\left(I_{n-1}\right)\right)=\left(h_{1}, \ldots, h_{n-1}\right) .
$$

(This reflects the general fact that complete integrability is preserved by the reduction process).

Assume now that the Hamiltonian $F$ has a homoclinic orbit $(\bar{q}(t), \bar{p}(t))$ joining a saddle point $\left(q_{0}, p_{0}\right)$ to itself. (The case of heteroclinic orbits connecting different saddle points proceeds in the same way.) The Hamiltonian system for $L^{0}$ thus has an $(n-1)$-parameter family of invariant $(n-1)$ dimensional tori $T\left(h_{1}, \ldots, h_{n-1}\right)$ given by

$$
\begin{aligned}
& G_{j}\left(I_{j}\right)=h_{j}=\text { const }\left[\text { i.e., } I_{j}=l_{j}=G_{j}^{-1}\left(h_{j}\right)\right], \\
& \begin{array}{l}
\theta_{j}=\Omega_{j}\left(l_{j} \theta_{n}+\theta_{j}(0)(\bmod 2 \pi), \quad j=1, \ldots, n-1,\right. \\
\quad q=q_{0}, \quad p=p_{0} .
\end{array}
\end{aligned}
$$

Correspondingly, the system for $F$ has an $n$-parameter family of invariant tori $T\left(h_{1}, \ldots, h_{n}\right)$. Henceforth we write the (phase) constants of integration $\theta_{j}(0)$ as $\theta_{j}^{0} j=1, \ldots, n-1, n$.

The torus $T\left(h_{1}, \ldots, h_{n-1}\right)$ is connected to itself by the $n$ dimensional homoclinic manifold

$$
\begin{aligned}
& G_{j}\left(I_{j}\right)=h_{j}, \\
& \theta_{j}=\Omega_{j}\left(I_{j}\right) \theta_{n}+\theta_{j}^{0}, \quad j=1, \ldots, n-1, \\
& q=\bar{q}\left(\theta-\theta_{n}^{0}\right), \quad p=\bar{p}\left(\theta-\theta_{n}^{o}\right),
\end{aligned}
$$

where the phase constant $\theta_{n}^{0}$ associated with the "reduced" degree of freedom appears explicitly. This manifold consists of the coincident stable and unstable manifolds of the torus $T\left(h_{1}, \ldots, h_{n-1}\right)$; i.e.,

$$
W^{s}\left(T\left(h_{1}, \ldots, h_{n-1}\right)\right)=W^{u}\left(T\left(h_{1}, \ldots, h_{n-1}\right)\right)
$$

given by (2.12). See Fig. 1.
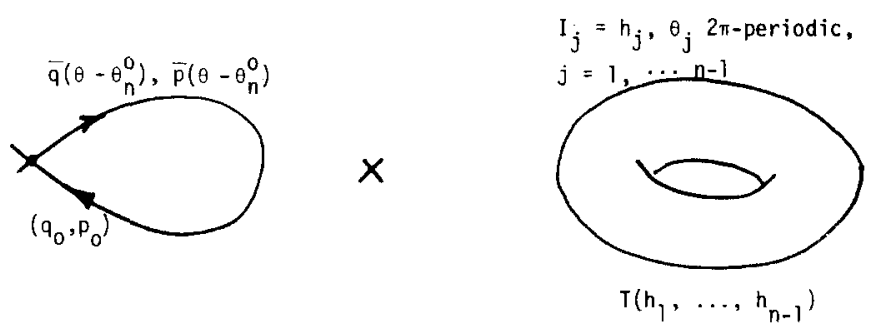

FIG. 1. The homoclinic orbit for the $F$ system $\times$ the invariant torus $T\left(h_{1}, \ldots, h_{n-1}\right)$ gives the homoclinic manifold of the torus.

For $\epsilon \neq 0$ the system (2.10) possesses a Poincaré map $P_{\epsilon}$ from (a piece of) $\left(q, p, \theta_{1}, \ldots, \theta_{n-1}, I_{1}, \ldots, I_{n-1}\right)$ space to itself, where $\theta_{n}$ goes through an increment of $2 \pi$, starting at some fixed value $\theta_{n}^{0}$ (which will be suppressed in the notation). The tori $T\left(h_{1}, \ldots, h_{n-1}\right)$ are invariant manifolds for $P_{0}$. In fact, these tori are isotropic submanifolds (i.e., the canonical 2 form $\omega$ vanishes on them), a fact we shall need later.

The program is to show that for $\epsilon \neq 0$ some of the tori persist and that their stable and unstable manifolds intersect transversely. To do this we shall invoke the KAM (Kolmogorov-Arnold-Moser) theory and Melnikov's method. The result will then be interpreted as Arnold diffusion.

Let us first discuss the invariant tori. The manifold obtained by setting $q=q_{0}, p=p_{0}$ is a $(2 n-2)$-dimensional normally hyperbolic invariant manifold, say $M_{0}$, for our Poincaré map $P_{0}$. Thus, for $\epsilon$ small, $M_{0}$ perturbs uniquely to an invariant manifold $M_{\epsilon}$ for $P_{\epsilon}$. The KAM theory now can be applied to the family of invariant tori $T\left(h_{1}, \ldots, h_{n-1}\right)$ on $M_{0}$. If the hypotheses of nondegeneracy and nonresonance hold ${ }^{8}$ then the torus $T\left(h_{1}, \ldots, h_{n-1}\right)$ will perturb to an invariant torus $T_{\epsilon}\left(h_{1}, \ldots, h_{n-1}\right)$ for $P_{\epsilon}$, for $\epsilon$ sufficiently small (depending upon the precise "degree" of nondegeneracy). Moreover, the proof shows that this torus is also isotropic. ${ }^{9}$ We note that the perturbed torus $T_{\epsilon}$ has the same frequencies $\Omega_{j}\left(h_{j}\right)$ as the unperturbed torus and thus the perturbed phase angles do not drift appreciably from the unperturbed ones. We use this fact below.

Although a set of positive measure of the perturbed tori persist near the original ones, the resonant tori containing continuous families of periodic motions generally break into finite sets of alternating elliptic and hyperbolic periodic orbits with associated homoclinic motions, as in Arnold [1978], ${ }^{8}$ (p. 397). The boundaries of the elliptic islands are conventionally drawn as homoclinic orbits of a flow: these actually belong to an associated averaged ( = canonically transformed) system. Restoration of the terms omitted in averaging leads to the prediction that these islands will, in turn, be surrounded by regions containing transverse homoclinic orbits (cf. Holmes ${ }^{4}$ ) but these regions are smaller than any power of $\epsilon$, since they can be removed by successive averaging operations. In fact such "stochastic layers" are generally exponentially small in $\epsilon$ and attempts to compute them by the Melnikov method necessitate a careful examination of errors. This will be the subject of a further publication; cf. Sanders [1980]. ${ }^{10}$

In the case of two degrees of freedom for which the 
unperturbed reduced system has a hyperbolic saddle point $x_{0}=\left(q_{0}, p_{0}\right)$, solutions of the perturbed system lying in the perturbed stable and unstable manifolds of the perturbed saddle point $x_{\epsilon}$ of the map $\mathrm{P}_{\epsilon}$ can be expanded in power series which converge uniformly in the intervals indicated:

$$
\begin{array}{ll}
W^{s} \cdot \bar{x}_{\epsilon}^{s}=\bar{x}\left(\theta-\theta^{0}\right)+\epsilon x_{1}^{s}\left(\theta, \theta^{0}\right)+O\left(\epsilon^{2}\right), & \theta \in\left[\theta^{0}, \infty\right), \\
W^{u} \cdot \bar{x}_{\epsilon}^{u}=\bar{x}\left(\theta-\theta^{0}\right)+\epsilon x_{1}^{u}\left(\theta, \theta^{0}\right)+O\left(\epsilon^{2}\right), & \theta \in\left(-\infty, \theta^{0}\right],
\end{array}
$$

where

$$
\bar{x}\left(\theta-\theta^{0}\right)=\left(\begin{array}{l}
\bar{q}\left(\theta-\theta^{0}\right) \\
\bar{p}\left(\theta-\theta^{0}\right)
\end{array}\right)
$$

is the unperturbed homoclinic orbit. (Recall that the periodic variable $\theta$ has replaced time.) For details, see Holmes, ${ }^{4}$ Sanders, ${ }^{10}$ or Greenspan and Holmes. ${ }^{5}$ [Basically (2.13) follows from the fact that the perturbed solutions lie in manifolds of solutions forward- or backward-asymptotic to the perturbed saddle points.] Similarly, solutions lying in the perturbed invariant manifolds $W^{s}\left(T_{\epsilon}\right), W^{u}\left(T_{\epsilon}\right)$ of a perturbed torus $T_{\epsilon}$ can be expanded in convergent power series in $\epsilon$ in such intervals, since the perturbed actions are $\epsilon$ close and the perturbed angles do not drift but remain close to the unperturbed angles on the tori. This result will be used implicitly in what follows.

The perturbed invariant manifolds $W^{s}\left(T_{\epsilon}\right)$ and $W^{u}\left(T_{\epsilon}\right)$ of the torus $T_{\epsilon}$ for the map $P_{\epsilon}$ are $n$-dimensional manifolds lying $C^{r}$ close to the unperturbed homoclinic manifold given by $(2.12)$, i.e.,

$$
F=\bar{h}, \quad I_{j}=l_{j}, \quad j=1, \ldots, n-1,
$$

where $\bar{h}$ is the energy of the homoclinic orbit for $F$. Now we are ready to give a criterion for the transversal intersection of $W^{s}\left(T_{\epsilon}\right)$ and $W^{u}\left(T_{\epsilon}\right)$. In order for the results to be applicable, it is useful to present the hypotheses in terms of data given for the original, rather than the reduced, system.

We consider a Hamiltonian system with $n+1(\geqslant 3)$ degrees of freedom of the form

$$
\begin{aligned}
& H^{\epsilon}\left(q, p, \theta_{1}, \ldots, \theta_{n}, I_{1}, \ldots, I_{n}\right) \\
& =F(q, p)+\sum_{i=1}^{n} G_{i}\left(I_{i}\right) \\
& \quad+\epsilon H^{1}\left(q, p, \theta_{1}, \ldots, \theta_{n}, I_{1}, \ldots, I_{n}\right) .
\end{aligned}
$$

Introduce the following assumptions and terminology:

(H1) $F$ contains a homoclinic orbit $(\bar{q}(t), \bar{p}(t))$ connecting a saddle point $\left(q_{0}, p_{0}\right)$ to itself. Let $\bar{h}$ be the energy of this orbit.

(H2) $\Omega_{j}\left(I_{j}\right)=G_{j}^{\prime}\left(I_{j}\right)>0$ for $j=1, \ldots, n$.

Let $h>h$ and let the unperturbed homoclinic manifold be filled with an $n$-parameter family of orbits given by $\left(\bar{q}, \bar{p}, \theta_{1}, \ldots, \theta_{n}, I_{1}, \ldots, I_{n}\right)=\left(\bar{q}(t), \bar{p}(t), \Omega_{1}\left(I_{1}\right) t+\theta_{1}^{0}, \ldots, \Omega_{n}\left(I_{n}\right) t\right.$ $\left.+\theta_{n}^{0}, I_{1}, \ldots, I_{n}\right)$. Pick one such orbit and let $\left\{F, H^{1}\right\}$ denote the $(q, p)$ Poisson bracket of $F(q, p)$ and $H^{1}\left(q, p, \theta_{1}, \ldots, \theta_{n}, I_{1}, \ldots, I_{n}\right)$ evaluated on this orbit. Similarly, let $\left\{I_{k}, H^{1}\right\}=-\partial H^{1} / \partial \theta_{k}, k \equiv 1, \ldots, n-1$ be evaluated on this orbit. Define the Melnikov Vector $M(\theta)$

$=\left(M_{1}, \ldots, M_{n-1}, M_{n}\right)$ by

$$
\begin{aligned}
& M_{k}\left(\theta_{1}^{0}, \ldots \theta_{n}^{0}, h, h_{1}, h_{2}, \ldots, h_{n-1}\right) \\
& \quad=\int_{-\infty}^{\infty}\left\{I_{k}, H^{1}\right\} d t, \quad k=1, \ldots, n-1 \\
& M_{n}\left(\theta_{1}^{0}, \ldots, \theta_{n}^{0}, h, h_{1}, h_{2}, \ldots, h_{n-1}\right) \\
& \quad=\int_{-\infty}^{\infty}\left\{F, H^{1}\right\} d t .
\end{aligned}
$$

(We note that $h_{n}=h-\bar{h}-\sum_{j=1}^{n} \bar{L}_{j}^{1} ; I_{n}$ and $h_{n}$ do not explicitly enter the calculations, since $I_{n}$ is eliminated by the reduction process; we also note that these integrals need not be absolutely convergent, but we do require conditional convergence.)

(H3) Assume that the constants $G_{j}\left(I_{j}\right)=h_{j} j=1, \ldots, n$, are chosen so that the unperturbed frequencies $\Omega_{1}\left(I_{1}\right), \ldots, \Omega_{1}\left(I_{n}\right)$ satisfy the nondegeneracy conditions [i.e., $\left.\Omega_{j}^{\prime}\left(I_{j}\right) \neq 0, j=1, \ldots, n-1\right]$ and the nonresonance conditions mentioned above (cf. Arnold, ${ }^{8}$ Appendix 8).

(H4) Assume that the multiply $2 \pi$-periodic Melnikov vector $M: \mathbb{R}^{n} \rightarrow \mathbb{R}^{n}$ (which is independent of $\epsilon$ ) has at least one transversal zero; i.e., there is a point $\left(\theta_{1}^{0}, \ldots, \theta_{n}^{0}\right)$ for which

$$
M\left(\theta_{1}^{0}, \ldots, \theta_{n}^{0}\right)=0
$$

but

$$
\operatorname{det}\left[D M\left(\theta_{1}^{0}, \ldots, \theta_{n}^{0}\right)\right] \neq 0,
$$

where $D M$ is the $n \times n$ matrix of partial derivatives of $M_{1}, \ldots, M_{n}$ with respect to $\theta_{1}^{0}, \ldots, \theta_{1}^{n}$, the initial phases of the orbit.

Here is our main theoretical result.

Theorem 2.1: If conditions (H1)-(H4) hold for the system (2.15) then, for $\epsilon$ sufficiently small, the perturbed stable and unstable manifolds $W^{s}\left(T_{\epsilon}\right)$ and $W^{u}\left(T_{\epsilon}\right)$ of the perturbed torus $T_{\epsilon}$ intersect transversally. (See Fig. 2.)

Remark: The conclusions imply that the perturbed system has no analytic integrals other than the total energy $H^{\epsilon}$ and, for $n+1 \geqslant 3$, that Arnold diffusion occurs. This is dis-

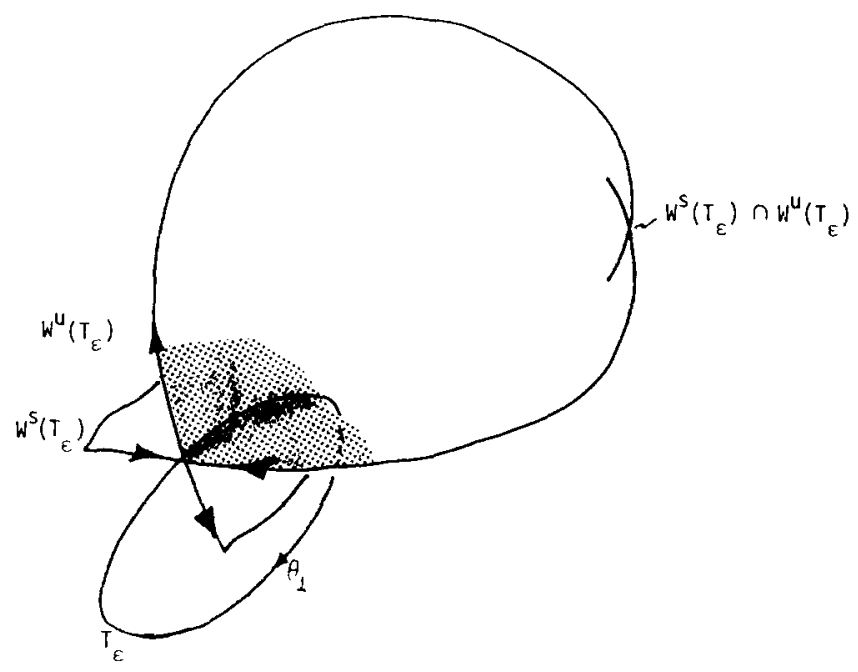

FIG. 2. The stable and unstable manifolds of the invariant torus $T_{\epsilon}$ for the Poincare map in the reduced space for a system with three degrees of freedom. This figure occurs in $\left(q, p, \theta_{1}, I_{1}\right)$ space [one dimension $\left(I_{1}\right)$ is suppressed], in a $\theta_{2}^{\circ}=$ fixed cross section for fixed total energy $h$. 
cussed in the next section.

Proof: First we notice that the brackets of the original functions project to corresponding brackets of the reduced system:

$$
\left\{L^{0}, L^{1}\right\}=\frac{1}{\left[\Omega_{n}\left(I_{n}\right)\right]^{2}}\left\{F, H^{1}\right\}
$$

and

$$
\left\{I_{k}, L^{1}\right\}=-\frac{1}{\Omega_{n}\left(I_{n}\right)}\left\{I_{k}, H^{1}\right\}, \quad k=1, \ldots, n-1
$$

(see Holmes and Marsden, ${ }^{1}$ Prop. 3.1).

We next wish to relate these brackets to a vector measuring the distance between the perturbed stable and unstable manifolds.

Consider the suspended system in

$\left(q, p, \theta_{1}, \ldots, \theta_{n-1}, I_{1}, \ldots, I_{n-1}\right)$ space. Pick a transversal $\Sigma_{\theta^{\circ}}$ to the unperturbed homoclinic manifold $W^{s}(T)=W^{u}(T)$ in $\left(q, p, \theta_{1}, \ldots, \theta_{n-1}, I_{1}, \ldots, I_{n-1}\right)$ space at the point $\left(\bar{q}(0), \bar{p}(0), \theta_{1}^{0}, \ldots, \theta_{n-1}^{0}, I_{1}, \ldots, I_{n-1}\right)$ and at "time" $\theta_{n}^{0}$. Now for $\epsilon$ sufficiently small, $W^{s}\left(T_{\epsilon}\right)$ and $W^{u}\left(T_{\epsilon}\right)$ intersect $\Sigma_{\theta^{\circ}}$ in unique points in $\left(q, p, \theta_{1}, \ldots, \theta_{n-1}, I_{1}, \ldots, I_{n-1}\right)$ space, which we denote

$$
x_{\epsilon}^{s}\left(\theta^{0}, \theta_{n}^{0}\right) \text { and } x_{\epsilon}^{u}\left(\theta^{0}, \theta_{n}^{0}\right) \text {. }
$$

The unique trajectories in $\left(q, p, \theta_{1}, \ldots, \theta_{n-1}, I_{1}, \ldots, I_{n-1}, \theta_{n}\right)$ space with these points as initial conditions and time $\theta_{n}$ will be denoted

$$
x_{\epsilon}^{s}\left(\theta^{0}, \theta_{n}\right) \text { and } x_{\epsilon}^{u}\left(\theta^{0}, \theta_{n}\right) .
$$

As in Holmes and Marsden, ${ }^{6}$ a measure of the distance between these vectors and the tangent to $W^{s}(T)=W^{u}(T)$ in the " $\theta_{n}$ direction," i.e., the direction of $X_{L}$ ", the Hamiltonian vector field of the unperturbed dynamics, is provided by the symplectic form $\omega$. Let

$\Delta_{\epsilon, n}\left(\theta_{n}, \theta_{n}^{\circ}\right)=\omega\left(X_{L^{0}}, x_{\epsilon}^{s}-x_{\epsilon}^{u}\right)=\Delta_{\epsilon, n}^{+}-\Delta_{\epsilon, n}^{-}+O\left(\epsilon^{2}\right)$.

Now as $\theta_{n} \rightarrow+\infty, x_{\epsilon}^{s} \rightarrow T_{\epsilon}$ and as $\theta_{n} \rightarrow-\infty, x_{\epsilon}^{\mu} \rightarrow T_{\epsilon}$ so, as in Holmes and Marsden ${ }^{6}$ Lemma 5, we obtain

$$
\begin{aligned}
\Delta_{\epsilon, n}\left(\theta_{n}^{0}\right)= & \Delta_{\epsilon, n}\left(\theta_{n}^{0}, \theta_{n}^{0}\right) \\
& -\frac{\epsilon M_{n}\left(\theta_{1}^{0}, \ldots, \theta_{n-1}^{0}, \theta_{n}^{0}\right)}{\left[\Omega\left(I_{n}\right)\right]^{2}}+O\left(\epsilon^{2}\right) .
\end{aligned}
$$

Note that the integrals (2.16) are well defined since one integrates forward along the stable manifold and backward along the unstable manifold [cf. Eq. (2.13)].

A crucial feature of this calculation is the fact that $\Delta_{\epsilon, n}^{+}\left(\theta_{n}, \theta_{n}^{0}\right) \rightarrow 0$ as $\theta_{n} \rightarrow \pm \infty$ since $\omega$ vanishes identically on $T$. This holds as follows. The invariant tori are isotropic and $\Delta_{\epsilon, n}^{+}\left(\theta_{n}, \theta_{n}^{0}\right)=\omega\left(X_{L^{u}}, \boldsymbol{\epsilon} X_{1}^{s}\right)$ where $X_{L^{0}}$ and $x_{1}^{s}$ are evaluated on the unperturbed homoclinic manifold and $x_{1}^{5}$ is the solution of the first variation equation. Since $X_{L^{\prime}}$ is tangent to $T$, $x_{1}^{s}$ necessarily approaches a tangent to $T$, so as $T$ is isotropic, $\Delta \underset{\epsilon, n}{+} \rightarrow 0$. We note that in this context the perturbed torus $T_{\epsilon}$ may move [by $O(\epsilon)]$ and need not remain fixed as in the special case treated by Arnold ${ }^{2}$ or as in Melnikov's " paper (cf. Holmes ${ }^{4}$ ).

Thus, $\boldsymbol{M}_{n}\left(\theta_{1}^{0}, \ldots, \theta_{n-1}^{0}, \theta_{n}^{0}\right)$ measures the leading non- trivial component of the distance between $W^{s}\left(T_{\epsilon}\right)$ and $W^{u}\left(T_{\epsilon}\right)$ (up to a constant) in a direction transverse to the "dynamic" variable $\theta_{n}$. Likewise, $M_{i}(i=1, \ldots, n-1)$ measures the distance between $W^{s}\left(T_{\epsilon}\right)$ and $W^{u}\left(T_{\epsilon}\right)$ in the direction transverse to the generator of the $\theta_{i}$ variable. The theorem now follows from these facts.

Remarks: 1. One can, of course, permute which of the action-angle variables is used for the reduction procedure. The remaining oscillators must satisfy the KAM nonresonance and nondegeneracy conditions.

2 . Gruendler ${ }^{12}$ has treated the $2 n$-dimensional, periodically forced case in which one also has an $n$-parameter family of unperturbed homoclinic orbits but in which they are homoclinic orbits to a hyperbolic saddle point $x$ and $\operatorname{dim} W^{s}(x)=\operatorname{dim} W^{u}(x)=n$. Again one obtains a generalized $n$ vector of Melnikov functions each depending upon $n$ arguments, one of which is the section time $\left(\theta_{n}^{0}\right)$ and the remaining $n-1$ of which serve to parametrize the family of orbits. The manifolds $W^{s}(x)$ and $W^{u}(x)$ are both necessarily isotropic, so one can proceed in a way analogous to that here. However, no KAM theory is needed and ordinary horseshoes rather than Arnold diffusion occur. Gruendler applies the theory to the case of a periodically forced spherical pendulum.

3. The theorem can be somewhat generalized. For example, many integrable systems do not decompose precisely as assumed in the form $F(q, p)+\sum_{j=1}^{n} G_{j}\left(I_{j}\right)$ and one sometimes finds that the unperturbed "frequencies," $\Omega_{j}=\partial G_{j} / \partial I_{j}$, also depend upon $(q, p)$. If this occurs, and $\Omega_{j}\left(I_{j}, q, p\right)$ is not constant on the unperturbed manifold, then it must be incorporated into the Poisson brackets [cf. Eqs. (2.17) and (2.18)]. This situation will be dealt with in Holmes and Marsden. ${ }^{\text {? }}$

4. Alan Weinstein has pointed out that even without hypothesis ( $\mathrm{H} 4)$, the stable and unstable manifolds of the perturbed torus $T_{\epsilon}$ must intersect. This comes about as follows. As in the standard Melnikov analysis (Holmes and Marsden), ${ }^{6}$ pick a $2 n$-dimensional cross section $\Sigma_{\theta_{n}^{\prime} \text {. The sta- }}$ ble and unstable manifolds $T_{\epsilon}$ for the associated Poincaré map, $W^{s}\left(T_{\epsilon}\right)$ and $W^{u}\left(T_{\epsilon}\right)$, are Lagrangian submanifolds of $\Sigma_{\theta_{n}^{\text {n }}}$ which are coincident at $\epsilon=0$. Lagrangian intersection theory (Arnold ${ }^{13}$ and Weinstein ${ }^{14}$ ) shows that the perturbed manifolds must intersect. This observation generalizes one of McGehee and Meyer. ${ }^{15}$ It follows that the hypothesis $(H 4)$ holds for generic perturbation terms $H^{1}$. However, condition (H4) allows one to check transversality in specific cases.

5. In contrast to our results, Easton and $\mathrm{McGehee}^{16}$ use Moser's ${ }^{17}$ fixed point theorem to show that some homoclinic orbits in a model system survive under special perturbations. Alan Weinstein points out that, similarly, at least two homoclinic orbits survive perturbations of the spherical pendulum's $S^{\prime}$ family of homoclinic orbits.

\section{NONINTEGRABILITY AND ARNOLD DIFFUSION}

If the stable and unstable manifolds $W^{s}(\Lambda), W^{u}(\Lambda)$ of a hyperbolic invariant set $\Lambda$ intersect transversely then it follows from the lambda lemma (Palis, ${ }^{18}$ Newhouse ${ }^{19}$ ) that $W^{s}(\Lambda)$ accumulates on itself and $W^{u}(\Lambda)$ accumulates on it- 
self. A similar result holds for the invariant tori in the present case (cf. Arnold, ${ }^{2}$ Theorem 1); more precisely, if $\Delta_{1} \subset W^{\mu}\left(T_{\epsilon}\right)$ is an $n$-dimensional neighborhood of a transverse homoclinic point $x \in W^{u}\left(T_{\epsilon}\right) \cap W^{s}\left(T_{\epsilon}\right)$, and $\Delta_{2} \subset W^{u}\left(T_{\epsilon}\right)$ is any open disk, then there are points of $\cup P_{n>0}^{n}\left(\Delta_{1}\right)$ lying arbitrarily close to $\Delta_{2}$. Such a torus $T_{\epsilon}$ is said to be a transition torus. The torus is said to lie in a transition chain of transition tori $T_{\epsilon}^{1}, T_{\epsilon}^{2}, \ldots, T_{\epsilon}^{k}$ if the unstable manifold $W^{\mu}\left(T_{\epsilon}^{j}\right)$ of the $j$ th torus transversely intersects the stable manifold of the $(j+1)$ st. This holds in our case since, by KAM theory, the set of "sufficiently irrational" tori preserved when $\epsilon \neq 0$ has measure $\mu(\epsilon) \rightarrow 1$ as $\epsilon \rightarrow 0$ (it is, in fact, a Cantor set). Thus, for sufficiently small $\epsilon$ one can find tori $T_{\epsilon}^{j}, T_{\epsilon}^{j+1}$ which are, along with their stable and unstable manifolds, arbitrarily $C^{r}$ close away from the torus and these manifolds have large "oscillations" near the torus as in Holmes and Marsden, ${ }^{1}$

Fig. B.1. It follows that if $W^{u}\left(T_{\epsilon}^{j}\right)$ intersects $W^{s}\left(T_{\epsilon}^{j}\right)$ transversely it must also intersect $W^{s}\left(T_{\epsilon}^{j+1}\right)$ transversely. Applying the same argument to $T_{\epsilon}^{j+1}, T_{\epsilon}^{j+2}, \ldots$, one constructs a transition chain. Orbits lying in $W^{u}\left(T_{\epsilon}^{j}\right)$ therefore accumulate on $W^{u}\left(T_{\epsilon}^{k}\right)$ for $k \geqslant j$ and these orbits and nearby ones provide a mechanism by which solutions can "diffuse" from the neighborhood of the torus to any other in the transition chain. (cf. Arnold, ${ }^{2}$ Theorem 2). An argument analogous to that above shows that $W^{u}\left(T_{\epsilon}^{j+1}\right)$ intersects $W^{s}\left(T_{\epsilon}^{j}\right)$ and thus that diffusion can take place in both directions along the chain. Note, however, that the length of the chain is generally governed by the perturbation strength $\epsilon$, since as $\epsilon$ increases the set of perturbed tori generally diminishes.

The mechanism outlined above, which we attempt to portray in Fig. 3, is the basis for Arnold diffusion. Clearly it can only occur in systems with three or more degrees of freedom $(n \geqslant 2)$, since the unperturbed $2 n$-dimensional reduced Poincaré map must admit continuous families of tori connected by smooth homoclinic manifolds, and this cannot occur in two dimensions. For more information, numerical examples, and physical insights, see Chirikov ${ }^{20}$ and Lieberman. ${ }^{21}$ The main physical consequence of diffusion is that (given sufficient time) energy can be transferred back and forth in relatively large amounts between distinct physical

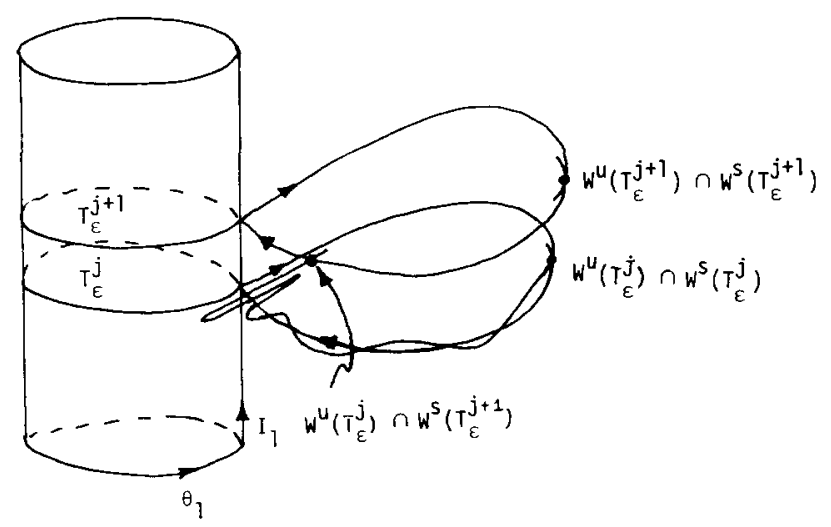

FIG. 3. Intersections of manifolds and Arnold diffusion in a three degree of freedom system. The Poincaré section $\theta_{2}=\theta_{2}^{0}$ is shown on the energy surface $H^{\epsilon}=h$. components or vibration modes of the system. Moreover this transfer of energy will typically take place in an irregular manner, in contrast to the regular quasiperiodic energy transfer occuring between modes in linear or other integrable systems.

Thus, in contrast to the two-degree-of-freedom case, in which the sufficiently irrational invariant tori, preserved for small perturbations, serve as boundaries to regions of homoclinic (chaotic) motions in the three-dimensional total-energy manifold, in systems with three or more degrees of freedom the solutions can diffuse from torus to torus along transition chains; the $n$ tori which are preserved do not bound regions of $2 n+1$ space for $n \geqslant 2$. In our case, since two-way transition chains can be chosen, we can find periodic motions of arbitrarily high period close to such chains, just as in the standard two-dimensional horseshoe example. The density of the set of such motions and the dense orbit accompanying them guarantees nonexistence of any additional analytic integrals other than the total energy $H^{\epsilon}$. (In fact one sees that such dense orbits exist within neighborhoods of any transverse homoclinic orbits connecting a torus to itself, without invoking the idea of diffusion.)

The presence of a small amount of noise in a system is believed to "stabilize" in some sense the occurrence of Arnold diffusion, in the same way that noise often "stabilizes" or "makes visible" horseshoes (cf. Holmes and Marsden. ').

\section{AN EXAMPLE:THE SIMPLE PENDULUM COUPLED TO TWO OSCILLATORS}

We illustrate the theory developed above with a generalization of our earlier two-degree-of-freedom pendulumoscillator model (Holmes and Marsden [1981] ${ }^{1}$ ). Consider a simple pendulum linearly coupled to two nonlinear oscillators. For simplicity we assume that the oscillators are identical (this is not important) and that their Hamiltonians can be expressed as $G\left(\left(x_{i}^{2}+y_{i}^{2}\right) / 2\right)$ or, equivalently, in action-angle coordinates as

with

$$
G\left(I_{i}\right), \quad i=1,2,
$$

$$
\Omega\left(I_{i}\right)=\frac{\partial G}{\partial I_{i}}\left(I_{i}\right) \neq 0 \quad \text { for } I_{i}>0
$$

and

$$
\Omega^{\prime}\left(I_{i}\right)=\frac{\partial^{2} G}{\partial I_{i}^{2}}\left(I_{i}\right) \neq 0
$$

[cf. Eqs. (2.3) and condition (H2) of Sec. 2]. Elimination of either $I_{1}$ or $I_{2}$ by reduction is then possible. For definiteness, we shall assume that $I_{2}$ is removed. Our assumption of the form $G\left(\left(x_{i}^{2}+y_{i}^{2}\right) / 2\right)$ is merely for computational convenience, since more "realistic" anharmonic oscillators lead to Hamiltonians $G\left(I_{i}\right)$ expressed in terms of, for example, elliptic functions (cf. Greenspan and Holmes ${ }^{5}$ ).

The system to be studied has the Hamiltonian

$$
\begin{aligned}
H^{\epsilon}= & p^{2} / 2-\cos q+G_{1}\left(I_{1}\right)+G\left(I_{2}\right) \\
& +(\epsilon / 2)\left[\left(\left(2 I_{1}\right)^{1 / 2} \sin \theta_{1}-q\right)^{2}+\left(\left(2 I_{2}\right)^{1 / 2} \sin \theta_{2}-q\right)^{2}\right] .
\end{aligned}
$$


The unperturbed orbits in the homoclinic manifold are given by

$$
\left(\bar{q}, \bar{p}, \theta_{1}, \theta_{2}, I_{1}, I_{2}\right)=( \pm 2 \arctan (\sinh t), \pm 2 \operatorname{sech} t,
$$$$
\left.\Omega\left(l_{1}\right) t+\theta_{1}^{0}, \Omega\left(l_{2}\right) t+\theta_{2}^{0}, l_{1}, l_{2}\right)
$$

where $h>1$ is the total energy and $\bar{h}=1$ the energy of the homoclinic orbit, and $G_{1}\left(l_{1}\right)=h_{1}, G_{2}\left(l_{2}\right)=h-1-h_{1}$. Assumptions $H(1)$ and $H(2)$ of Theorem 3.1 are therefore satisfied and, in view of (4.2a) and (4.2b) we can pick $h$ and $h_{1}$ so that the nonresonance conditions necessary for application of the KAM theorem are met. To check the final assumption we compute the Poisson brackets $\left\{I_{1}, H^{1}\right\}$ and $\left\{F, H^{1}\right\}$. From (4.3) we have

$$
\left\{I_{1}, H^{1}\right\}=-\frac{\partial H^{1}}{\partial \theta_{1}}=-\left(\left(2 I_{1}\right)^{1 / 2} \sin \theta_{1}-q\right)\left(2 I_{1}\right)^{1 / 2} \cos \theta_{1}
$$

and

$$
\begin{aligned}
\left\{F, H^{1}\right\}= & \frac{\partial F}{\partial q} \frac{\partial H^{1}}{\partial p}-\frac{\partial F}{\partial p} \frac{\partial H^{1}}{\partial p} \\
= & \sin q \cdot 0-p\left[-\left(\left(2 I_{1}\right)^{1 / 2} \sin \theta_{1}-q_{1}\right)\right. \\
& \left.-\left(\left(2 I_{2}\right)^{1 / 2} \sin \theta_{2}-q\right)\right] \\
= & p\left[\left(2 I_{1}\right)^{1 / 2} \sin \theta_{1}+\left(2 I_{2}\right) \sin \theta_{2}-2 q\right] .
\end{aligned}
$$

Thus, using (4.4) we have

$$
\begin{aligned}
M_{1}\left(\theta_{1}^{0}, \theta_{2}^{0}, h, h_{1}\right) \\
=\int_{-\infty}^{\infty}-\left[\left(2 l_{1}\right)^{1 / 2} \sin \left(\Omega\left(l_{1}\right) t+\theta_{1}^{0}\right)\right. \\
\quad \mp 2 \arctan (\sinh t)] \\
\quad \times\left(2 l_{1}\right)^{1 / 2} \cos \left(\Omega\left(l_{1}\right) t+\theta_{1}^{0}\right) d t
\end{aligned}
$$

and

$$
\begin{aligned}
& M_{2}\left(\theta_{1}^{0}, \theta_{2}^{0}, h, h_{1}\right) \\
& =\int_{-\infty}^{\infty} \pm 2 \operatorname{sech} t\left[\left(2 l_{1}\right)^{1 / 2} \sin \left(\Omega\left(l_{1}\right) t+\theta_{1}^{0}\right)\right. \\
& \left.\quad+\left(2 l_{2}\right)^{1 / 2} \sin \left(\Omega\left(l_{2}\right) t+\theta_{2}^{0}\right) \mp 4 \arctan (\sinh t)\right] d t .
\end{aligned}
$$

Noting that the integrals of products of odd and even functions vanish over the infinite domain and taking the positive branch of the homoclinic manifold, these two functions become

$$
\begin{gathered}
M_{1}=2\left(2 l_{1}\right)^{1 / 2}\left[\int_{-\infty}^{\infty} \arctan (\sinh t) \sin \left(\Omega\left(l_{1}\right) t\right) d t\right] \sin \theta_{1}^{0}, \\
M_{2}=2\left(2 l_{1}\right)^{1 / 2}\left[\int_{-\infty}^{\infty} \operatorname{sech} t \cos \left(\Omega\left(l_{1}\right) t\right) d t\right] \sin \theta_{1}^{0} \\
\quad+2\left(2 l_{2}\right)^{1 / 2}\left[\int_{-\infty}^{\infty} \operatorname{sech} t \cos \left(\Omega\left(l_{2}\right) t\right) d t\right] \sin \theta_{2}^{0} .(4.7)
\end{gathered}
$$

For brevity we write

$$
\Omega\left(l_{1}\right)=\omega_{1}, \quad \Omega\left(l_{2}\right)=\omega_{2} .
$$

To evaluate the first conditionally convergent integral we choose, for computational convergence, the limits as follows:

$$
\begin{aligned}
\lim _{N \rightarrow \infty} \int_{-N \pi / \omega_{1}}^{N \pi / \omega_{1}} \arctan (\sinh t) \sin \omega_{1} t d t \\
=\lim _{N \rightarrow \infty}\left\{-\left.\frac{1}{\omega_{1}} \arctan (\sinh t) \cos \omega_{1} t\right|_{-N \pi / \omega_{1}} ^{N \pi / \omega_{1}}\right. \\
\left.\quad+\frac{1}{\omega_{1}} \int_{-N \pi / \omega_{1}}^{N \pi / \omega_{1}} \frac{\cos \omega_{1} t}{1+\sinh ^{2} t} d t\right\} \\
=\frac{1}{\omega_{1}} \int_{-\infty}^{\infty} \operatorname{sech}^{2} t \cos \omega_{1} t d t \\
=\frac{1}{\omega_{1}}\left(\pi \omega_{1}\right) \operatorname{csch}\left(\frac{\pi \omega_{1}}{2}\right) .
\end{aligned}
$$

The final integral is obtained by the method of residues.

Similarly, we have

$$
\int_{-\infty}^{\infty} \operatorname{sech} t \cos \omega_{j} t d t=\pi \omega_{j} \operatorname{sech}\left(\frac{\pi \omega_{j}}{2}\right), \quad j=1,2,
$$

and thus

$$
\begin{aligned}
M_{1}= & 2 \pi\left(2 l_{1}\right)^{1 / 2} \operatorname{csch}\left(\frac{\pi \omega_{1}}{2}\right) \sin \theta_{1}^{0}, \\
M_{2}= & {\left[\left(2 l_{1}\right)^{1 / 2} \omega_{1} \operatorname{sech}\left(\frac{\pi \omega_{1}}{2}\right) \sin \theta_{1}^{0}\right.} \\
& \left.\quad+\left(2 l_{2}\right)^{1 / 2} \omega_{2} \operatorname{sech}\left(\frac{\pi \omega_{2}}{2}\right) \sin \theta_{2}^{0}\right] .
\end{aligned}
$$

One obtains a similar result (with an appropriate change of sign) on the negative branch of the manifold.

We therefore find zeroes when $\theta_{1}^{0}=k \pi, \theta_{2}^{0}=l \pi$ for all integers $l, k$ and it is easy to check that

$$
\begin{aligned}
& \operatorname{det} D M=\frac{\partial M_{1}}{\partial \theta_{1}^{0}} \frac{\partial M_{2}}{\partial \theta_{2}^{0}}-\left.\frac{\partial M_{1}}{\partial \theta_{2}^{0}} \frac{\partial M_{2}}{\partial \theta_{1}^{0}}\right|_{\substack{\theta_{1}^{\prime \prime}=k \pi \\
\theta_{2}^{0}=l \pi}} \\
& \pm 8 \pi^{2} \omega_{2}\left(l_{1} l_{2}\right)^{1 / 2} \operatorname{csch}\left(\frac{\pi \omega_{1}}{2}\right) \operatorname{sech}\left(\frac{\pi \omega_{2}}{2}\right) \neq 0 .
\end{aligned}
$$

Thus the final assumption is satisfied for suitable choices of $h$ and $h_{1}$ and we have

Theorem 4.1: For $\epsilon$ sufficiently small the Hamiltonian system (4.3) has a set of two-dimensional invariant tori of positive measure each of whose unstable manifolds intersects its stable manifold transversely. Moreover, a finite transition chain of such tori $T_{\epsilon}^{1}, \ldots, T_{\epsilon}^{m}$ can be chosen such that $W^{u}\left(T_{\epsilon}^{j}\right)$ intersects $W^{s}\left(T_{\epsilon}^{j+1}\right)$ transversely and $W^{u}\left(T_{\epsilon}^{j+1}\right)$ intersects $W^{s}\left(T_{\epsilon}^{j}\right)$ transversely, $j=1, \ldots, m-1$. Thus, orbits can be found which pass from the neighborhood of any torus $T_{\epsilon}^{k}$ to the neighborhood of any other torus $T_{\epsilon}^{l}$ in the chain. This situation obtains on every energy level $H^{\epsilon}=h>1$.

Remarks: 1. Arnold's ${ }^{2}$ example is similar to ours in some respects, but he employed explicit external forcing, taking a $t$-periodic two-degree-of-freedom system $H^{\epsilon}(q, p, \theta, I, t)$ rather than a three-degree-of-freedom autonomous system. This perturbation was further chosen to vanish on the tori, so that the perturbed tori lie in the same positions as the unperturbed tori. As we remarked in Sec. 3, this is not necessary since the bracket $\omega\left(X_{L_{0}}, X_{L_{1}}\right)=\left\{L^{0}, L^{1}\right\}$ vanishes on the unperturbed isotropic tori, and thus the integral of the Poisson bracket along the unperturbed orbits still provides a good measure of the separation of the perturbed manifolds. 
2. Although the theorem asserts that diffusion occurs on every energy level $h>1$, the latitude available for choice of $h_{1}$ and hence for satisfaction of the nonresonance conditions increases with $h$. Thus the "sufficiently small $\epsilon$ " approaches zero as $h \rightarrow 1$.

\section{CONCLUSIONS}

This paper and its companions (Holmes and Marsden $\left.^{1,7}\right)$, address the general question of perturbations of integrable multidimensional Hamiltonian systems. A particular area of interest is the development of a method for investigating the integrability of the perturbed problem, and for providing a qualitative description of orbits in phase space.

In the present paper we have combined a reduction technique with a vectorial version of Melnikov's ${ }^{11}$ method to establish the existence of Arnold diffusion in Hamiltonian systems with at least three degrees of freedom. This in turn implies that the system is nonintegrable in the classical sense: there are no analytic integrals other than the total energy. The method is applied to the specific case of a pendulum coupled to two nonlinear oscillators. It is shown that the stable and unstable manifolds of nonresonant tori that survive under a small perturbation intersect transversely. We briefly discuss how this enables points in phase space to diffuse.

\section{ACKNOWLEDGMENTS}

We thank Alan Weinstein for several helpful discussions and Allan Kaufman for suggesting a stimulating physical example.

'P. J. Holmes and J. E. Marsden, "Horseshoes in perturbations of Hamiltonian systems with two degrees of freedom," Comm. Math. Phys. 82, 524 (1982).

${ }^{2} \mathrm{~V}$. Arnold, "Instability of dynamical systems with several degrees of free- dom," Dokl. Akad. Nauk. SSSR 156, 9 (1964).

${ }^{3}$ S. N. Chow, J. K. Hale, and J. Mallet-Paret, "An example of bifurcation to homoclinic orbits," J. Diff. Equations 37, 351 (1980).

${ }^{4}$ P. Holmes, "Averaging and chaotic motions in forced oscillations,"

SIAM J. Appl. Math. 38, 68 (1980); 40, 167 (1980).

${ }_{5}^{5}$ B. Greenspan and P. J. Holmes, "Homoclinic Orbits, Subharmonics and Global Bifurcations in Forced Oscillators," in Non-linear Dynamics and Turbulence, edited by G. Barenblatt, G. Iooss, and D. D. Joseph (Pitman, New York, to appear)

${ }^{6} \mathrm{P}$. J. Holmes and J. E. Marsden, "A partial differential equation with infinitely many periodic orbits: chaotic oscillations of a forced beam," Arch. Rat. Mech. Anal. 76, 135 (1981).

'P. J. Holmes and J. E. Marsden, "Horseshoes and Arnold diffusion for Hamiltonian systems on Lie groups," Indiana U. Math. J. (to appear). ${ }^{8} \mathrm{~V}$. Arnold, Mathematical methods of classical mechanics, Springer Graduate Texts in Mathematics, No. 60 (Springer, New York, 1978).

${ }^{9} \mathrm{~J}$. Moser, "Stable and random motions in dynamical systems, with special emphasis on celestial mechanics," in Ann. Math. Studies, No. 77 (Princeton U. P., Princeton, N. J., 1973).

${ }^{10} \mathrm{~J}$. A. Sanders, "Note on the validity of Melnikov's method and averaging," Report \#139, Wiskundig Seminarium, Vrije Universiteit Amsterdam (1980) (preprint).

${ }^{11}$ V. K. Melnikov, "On the stability of the center for time-periodic perturbations," Trans. Moscow Math. Soc. 12, 1 (1963).

${ }^{12}$ J. Gruendler, Thesis, University of North Carolina (1981).

${ }^{13} \mathrm{~V}$. I. Arncld, "Sur une propiétè topologique des applications globelement canonique de la mécanique classique," C. R. Acad. Sci. Paris 26, 3719 (1965).

${ }^{14}$ A. Weinstein, Lagrangian submanifolds and Hamiltonian systems. Ann. Math. 98, 377 (1973).

${ }^{15} \mathrm{R}$. McGehee and $\mathrm{K}$. Meyer, "Homoclinic points of area preserving diffeomorphism," Am. J. Math 96, 409 (1974).

${ }^{16} \mathrm{R}$. W. Easton and R. McGehee, "Homoclinic phenomena for obits doubly asymptotic to an invariant three sphere," Indiana Univ. Math. J. 28, 211 (1979).

${ }^{17} \mathrm{~J}$. Moser, "A fixed point theorem in symplectic geometry," Acta Math. 141, 17 (1978).

${ }^{18}$ J. Palis, "On Morse-Smale dynamical systems," Topology 8, 385 (1969).

19 $\mathrm{S}$. Newhouse, "Lectures on dynamical systems," in Dynamical Systems, edited by J. Moser (Birkhauser, Boston, 1980), pp. 1-114.

2"B. V. Chirikov, "A universal instability of many dimensional oscillator systems," Phys. Rep. 52, 265 (1979).

${ }^{21}$ M. A. Lieberman, Arnold diffusion in Hamiltonian systems with three degrees of freedom," Ann. N. Y. Acad. Sci. 357, 119 (1980). 